World Maritime University

The Maritime Commons: Digital Repository of the World Maritime University

Conference Proceedings

2021

\title{
Adaptive training with cloud-based simulators in maritime education
}

Simen Hjellvik

University of South-Eastern Norway

Steven Mallam

University of South-Eastern Norway

Follow this and additional works at: https://commons.wmu.se/imla2021

Part of the Education Commons

\section{Recommended Citation}

Hjellvik, S. \& Mallam, S. (2021). Adaptive training with cloud-based simulators in maritime education. In Pazaver, A., Manuel, M. E., Bolmsten, J., Kitada, M., Bartuseviciene, I. (Eds.), Proceedings of the International Maritime Lecturers' Association. Seas of transition: setting a course for the future (pp. 179-190). World Maritime University. . http://dx.doi.org/10.21677/imla2021.21

This Paper is brought to you courtesy of Maritime Commons. Open Access items may be downloaded for noncommercial, fair use academic purposes. No items may be hosted on another server or web site without express written permission from the World Maritime University. For more information, please contact library@wmu.se. 


\title{
International Maritime Lecturers' Association (IMLA) 2021 Joint Conference with IMEC32, ICERS15 and INSLC21
}

\section{Adaptive training with cloud-based simulators in maritime education}

\author{
Simen Hjellvik (D) https://orcid.org/0000-0002-2338-4698 \\ Assistant Professor \& Doctoral Research Fellow \& Chief Engineer, University of South- \\ Eastern Norway, Horten, Norway, simen.hjellvik@usn.no
}

\author{
Steven Mallam (D) https://orcid.org/0000-0003-1713-2977 \\ Associate Professor, University of South-Eastern Norway, Horten, Norway, \\ steven.mallam@usn.no
}

\begin{abstract}
Maritime cloud-based simulation is an emerging technological development that creates a new condition for decentralized interaction where it's content and functionality mirrors traditional on-site-simulator software. This paper uses a quasi-experimental study to examine a training design that is adaptive to the trainee. The training goal is to deliver traditional learning outcomes of comprehension and familiarity with the operation of steering gear systems. The simulator training was administered through novel cloud-based simulator technology to a sample comprising of first year students in nautical sciences $(n=12)$ and marine engineering $(n=6)$ at the college and university level in Norway who had no previous education or operational level experience with steering gear systems in their respective programmes. All participants $(N=18)$ were first subjected to a knowledge acquisition phase of video conference lectures before conducting a simulator training scenario of a standardized pre-departure procedure. Data was collected from 3 sources: (1) a multiple-choice knowledge test, (2) programmed simulator performance indicators, and (3) the Self-Efficacy for Learning and Performance scale. Initial results show that the level of student's self-efficacy predicts the final training performance, and the level of knowledge prior to training is not significant for the outcome.
\end{abstract}

Keywords: Maritime Education and Training (MET), Simulator Training (ST), Cloud-Based Simulator (CBS), Virtual Reality (VR), Self-Efficacy, Engine Room Simulators (ERS).

\section{Introduction}

By provision of the International Convention on Standards of Training, Certification and Watchkeeping for Seafarers (STCW, 2017), the pedagogical structure of the competence development in professional maritime education and training (MET) incorporates both theory and practice through lecture-based education, simulation-based training, and on-board training. Cloud-based simulators (CBS) expands MET technologies as a potential alternative or supplemental solution to more well-established on-site campus simulators. CBS is a simulator technology that works on similar platforms as E-learning, where the simulator itself is located on a server which a user connects to through the internet. This creates new opportunities and challenges for how and where trainees, instructors and administrators access, interact and engage with the simulators and training content. CBS and decentralized learning removes, 
reduces or reorganizes the traditional supportive structures found in traditional on-site, inperson classroom and simulator laboratory interactions and collaborations between traineeinstructor and trainee-trainee peers. This emerging format for simulator training requires differing expectations, skills and motivation from trainees and instructors. The effect of selfefficacy is an individual characteristic that is important for self-regulatory learning and in effect, task performance (Zimmerman, 2008). Self-efficacy can be define as the trainee`s selfperception of their performance during repeated training (Kraiger et al., 1993). With a move towards decentralized learning and CBS solutions issues related to trainee self-efficacy, motivation and supportive structures during simulator exercises may become important for attaining learning outcomes.

This paper explores a CBS training scenario with substitutes for the loss of supportive structures of the on-site simulator training which traditionally includes a present peercolloquium and simulator instructors. Maritime simulator developers have been aware of the gap in technology for "personalized, immersive, mobile and accessible platforms" (Mallam et al., 2019) and the present development of CBS is an initial response to accommodate these features for optimized on-demand and asynchronous simulator training. An important aspect for asynchronous decentralized training is to provide adequate feedback to trainees throughout their CBS sessions. CBS exercises require programming for automated feedback and correctional instruction, including different levels of supportive structures and thus adapting to the trainee's level as the task complexity and goal is programmed to be alternatives selected by the trainee. From these concepts the study investigates the research question: How can CBS adapt to individual training needs?

To evaluate the sufficiency of the training design and administration, it is hypothesized that the training itself will be the major contribution to the overall task specific learning process.

Hypothesis 1 states: $\left(\mathrm{H}_{1}\right)$ Knowledge prior to training will be significant to the final training performance.

With the decentralized training delivery designed for this study the trainees themselves decide, based on their confidence, when they are ready to proceed from the training scenario to the test scenario. Hypothesis 2 states: $\left(\mathrm{H}_{2}\right)$ the level of self-efficacy will positively predict the final training performance.

The outcomes of the study could provide inspiration and preliminary research-based evidence for MET educators when administering decentralized simulator training for novice trainees.

\section{Theory}

\section{Learning Outcomes}

Lower level of cognitive learning outcomes, including acquiring fundamental declarative knowledge, traditionally precedes training. Establishing a knowledge foundation prior to taskspecific simulator training aid to not overwhelm the cognitive capacity of trainees for solving problems (Chernikova et al., 2020). Thus, simulator training develops both knowledge-based outcomes, as task-specific comprehension is necessary for the training, and skill-based outcomes, as technical skills is necessary for performing the task successfully. Declarative knowledge in the knowledge-acquisition phase is often evaluated by memory power tests which probe the accuracy and accessibility of retaining memory of the specific knowledge 
items, or by recall tests which probe the amount of knowledge acquired (Kraiger et al., 1993). Such knowledge tests, e.g., typical grade exams, should probe beyond reiteration and require application of knowledge to a context.

Skill-based outcomes involve an initial skill-acquisition in which declarative system knowledge is transformed into procedural knowledge as it is repeatedly and adequately applied with a goal orientation (Smith et al., 2019). Proceduralization is the outcome occurring when reproducing trained behaviour beyond the initial stage, and accumulates decomposed steps of the task in less error-prone performance (Kraiger et al., 1993). Just as variance in trainee knowledge is expected to be larger at the initial stage of the knowledge-acquisition phase, variance of trainees performance should converge at the final stage of the skill-acquisition phase and is in training research argued to be an indicator of training effectiveness(Bell et al., 2017).

\section{Self-Efficacy in Learning}

Self-efficacy is the perceived performance capability for a task, and by decomposing a task, as steps in a procedure, the training facilitates development of stronger perceptions of selfefficacy concurrent with the capability to perform (Kraiger et al., 1993). Self-efficacy is a skillbased learning outcome that shows numerous positive relations with performance and should be considered when designing training programmes, as greater confidence in one`s task capabilities empower resilience towards challenges when applying knowledge and skills (Bell et al., 2017; Ford et al., 1998; Kraiger et al., 1993). Furthermore, self-efficacy has been shown to be positively correlated with both performance goals and actual performance (Zimmerman, 2008).

In training, feedback is an essential element in the process of performance approximating the goal. It can enhance self-efficacy or have no positive effect at all, depending on the trainee`s reception (Hattie \& Timperley, 2016). How familiar the material is to the trainee impacts if they accept, modify, or reject feedback, thus the effect of knowledge on performance is contingent on connecting the new information to the trainee`s knowledge foundation (Hattie \& Timperley, 2016). Trainees with a low level of preceding knowledge benefit more from guiding instruction than self-regulation and reflection activities in comparison to trainees with higher preceding knowledge (Chernikova et al., 2020). If conceptualized as a unitary variable, the complexity of feedback can be adapted with increments as a response to the training progress with a transformation of the form in which the feedback is structured and administered (Figure 1). The objective of feedback is to eliminate the gap between the current performance and the training goal. In a successful self-regulatory learning process, the feedback loop will be dynamic and involve multiple cycles (Zimmerman, 2008). 


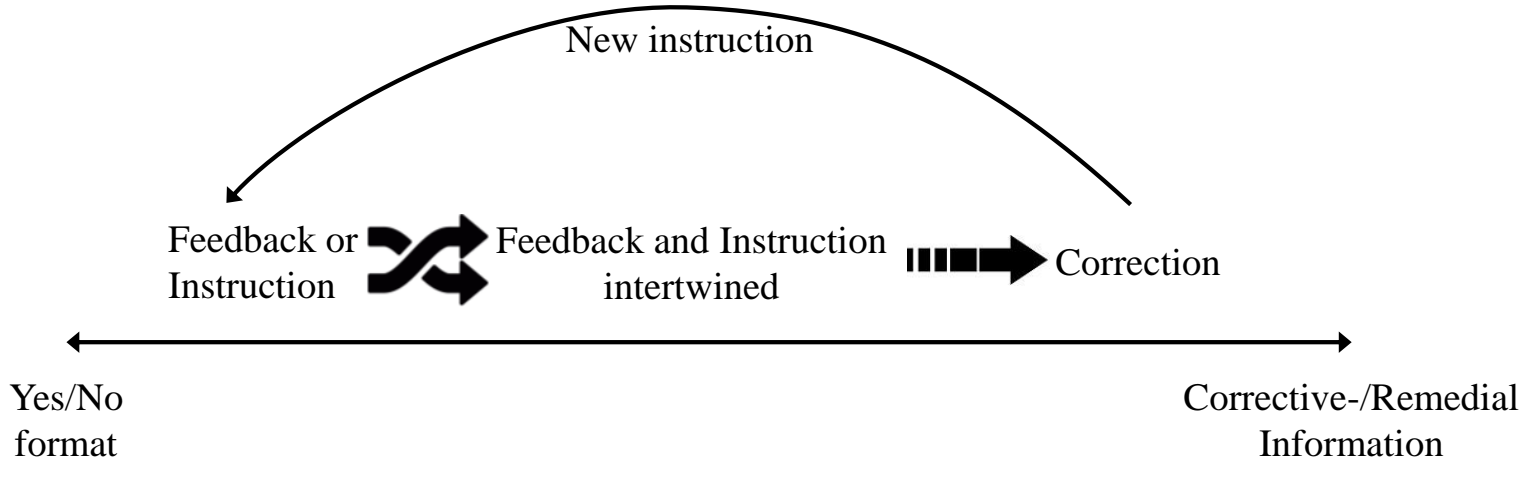

Feedback complexity

Figure 1. Feedback complexity model based on Hattie and Timperley (2016) and Kulhavy (1977)

\section{Decentralized Training Delivery}

Little is known to whom simulations are particularly useful and what supportive instructions is effective for the individual trainee (Chernikova et al., 2020). Thus, devising decentralized training and learning with a high level of learner control creates both challenges and opportunities. The social supportive structures in the traditional simulator training context, including instructor- and peer- support, will not be present with strictly asynchronous CBS configuration, which in general is a deficit, as learning is somewhat dependent on the environment of and around the training interaction. Decentralized platforms with a synchronous configuration do exist, where the instructor can monitor and communicate with the trainees in real-time. More important than the comparisons between technology effectiveness, research should focus on the pedagogical features and the conditions where technology-based training is likely to be effective (Bell et al., 2017). The opportunities for CBS training subsume generalized theory from training research where the trainee characteristics influencing performance should correspond with the design. Although real-time individual instructor-trainee feedback, i.e., synchronous interaction, is presently unavailable with this CBS platform, the technology allows programming exercises leveraging the theory on feedback, which generically can correspond with the procedure stages and task performance. Simulations that leverage the use of different mental modes and abilities (e.g., reasoning combined with motor skills) gains higher learning than simulations that require the involvement of less skills (Chernikova et al., 2020). Thus, the delivery of training can be adaptive to the trainee's knowledge- and performance level, and practical needs.

\section{Methods}

\section{Participants}

The sample $(N=18)$ were recruited from second semester first-year students enrolled in a Nautical Sciences $(n=12)$ and Marine Engineer $(n=6)$ programme at university and college levels in Norway. The study was integrated in their respective machinery courses, as the specific learning outcome is relevant for both disciplines. Collecting data was only conducted after written consent according to the approved Norwegian Centre for Research Data notification (no.753508). The average age of the sample was 23,7 years $(S D=5.39)$ and their prior maritime work experience was on average 2,9 years $(S D=3.31)$. 


\section{Cloud-Based Engine Room Simulator}

The cloud-based K-Sim Connect platform was used with the K-Sim Engine MAN 6S70ME-C SCC simulator. The simulator is a duplicate of the K-Sim Engine L11ME-SCC on-site desktop simulator model based on a Suez max crude oil carrier. The CBS platform allows simulator access from the individual trainee`s personal computers, requiring only an internet connection to operate, and is not contingent on an active simulator instructor. As such, the trainee is free to access the simulator at all hours from any location. The simulator interface operates on a $2 \mathrm{D}$ level (See Figure 2) where the machinery systems are replicated as line diagrams with components which can be manipulated. Components and parameter values in the system change according to the simulator programming or the trainee`s interaction, simulating the reallife system.

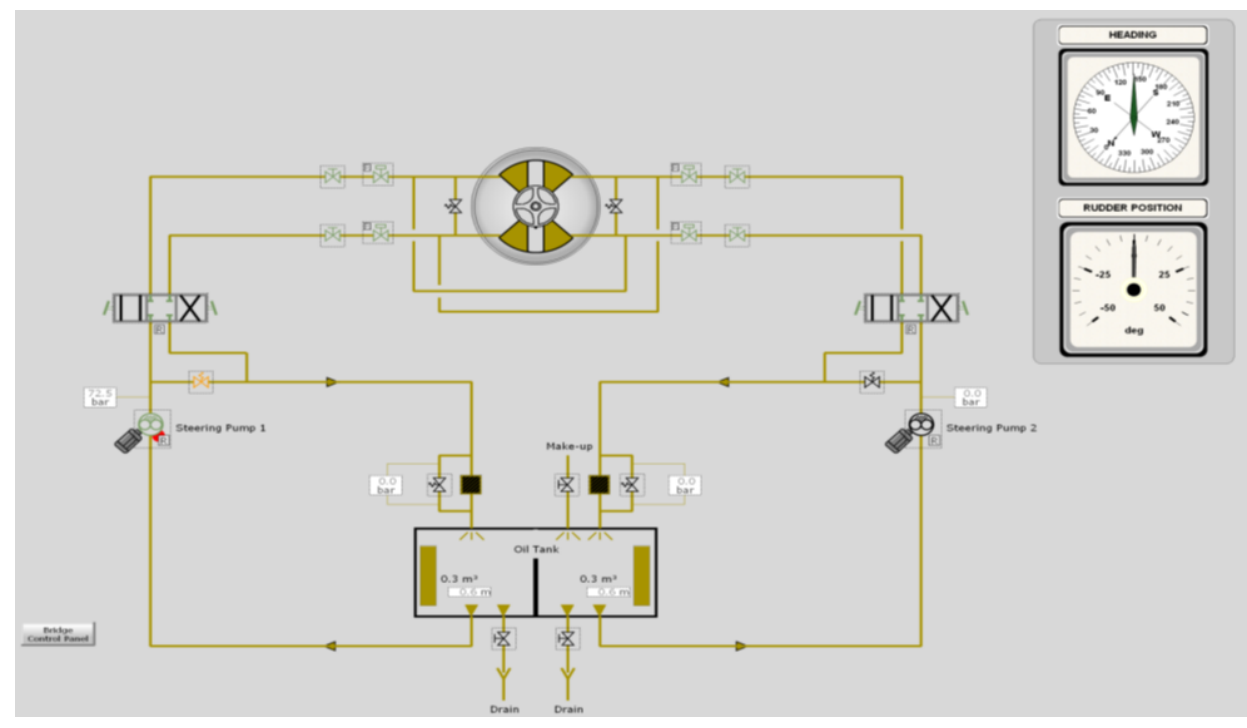

Figure 2. The simulator steering gear systems as presented to the trainee.

\section{Experimental Design and Procedure}

The experimental procedure is outlined in Figure 3.

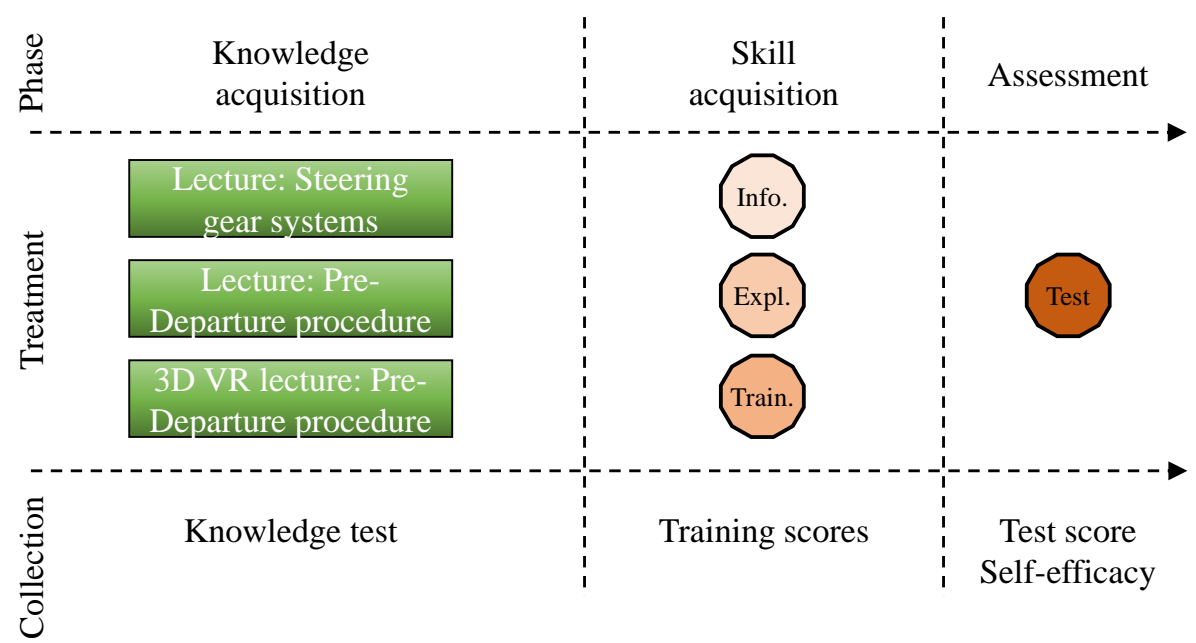

Figure 3: Experiment procedure and data collection 
The specific goal of the training was to perform a successful pre-departure procedure with the steering gear system, involving the tasks conducted locally in the steering gear room and remotely from the bridge. After completing the knowledge acquisition phase, the trainees were given access to the CBS and instructed to use the Information and Explore scenario to aggregate their knowledge foundation before performing the procedure in the Training scenario. It was possible for trainees to revisit these during training to improve performance and address any emerging knowledge gaps. No threshold defining a successful performance, nor any other external expectancy was defined for the trainees. The Training scenario was to be repeated until trainees were confident enough to progress to the final Test scenario.

\section{Knowledge Acquisition Phase}

To establish a declarative knowledge foundation the trainees were given three lectures:

- Lecture 1 (45 minutes): Focused on the different types and functionalities of steering gear systems through live video conference, provided generic system knowledge.

- Lecture 2 (45 minutes): Focused on the pre-departure procedure in the context of the simulator through live video conference, provided system specific and procedural knowledge.

- Lecture 3 (30 minutes): The instructor conducted the procedure in 3D virtual reality (VR) with audio voiceover lecturing (Figure 4), through a pre-recorded video lecture, providing visuospatial knowledge of the specific system to be trained. In this application the interaction with the simulator used a first-person view, as in most video games and all manipulations of components are animated in the virtual environment. The 3D VR module of the simulator was not available to the trainees through the CBS for practice, only the 2D interfaces as in Figure 2.

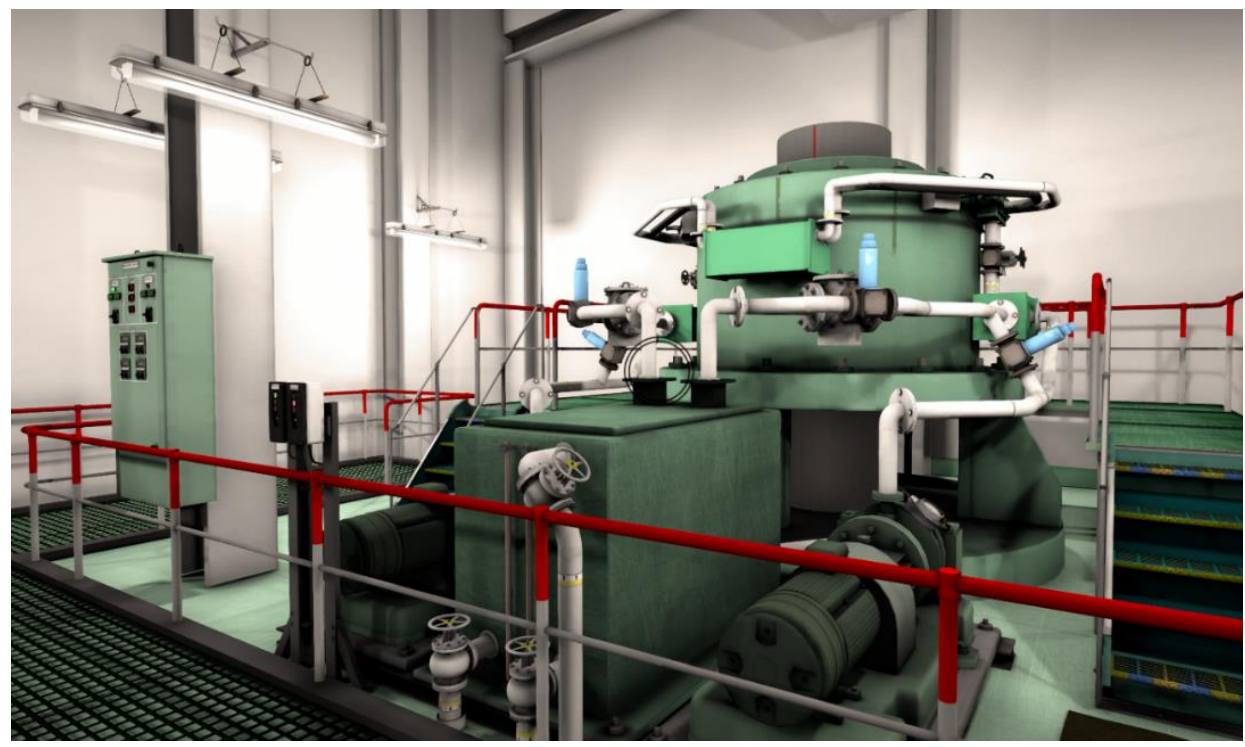

Figure 4. The simulator steering gear system as viewed through the 3D VR application. 


\section{Skill Acquisition Phase}

The K-Sim Neptune Instructor software was used to program the exercise and simulator metrics for data collection. The exercise was programmed for training and assessment purposes to give text responses to the trainee upon manipulation of variables in the simulator. The variable manipulations were comprised to represent independent or stepwise task achievement which was used to represent performance of the instructed task. This metric assessment was accumulated with positively- and negatively- weighted actions which accretes to a final assessment scale that is automatically shared with the trainee upon completion of the exercise. The exercise was programmed with three training levels and one test level as displayed in Figure 5. When starting the exercise, the trainees select the scenario alternative at their own discretion.

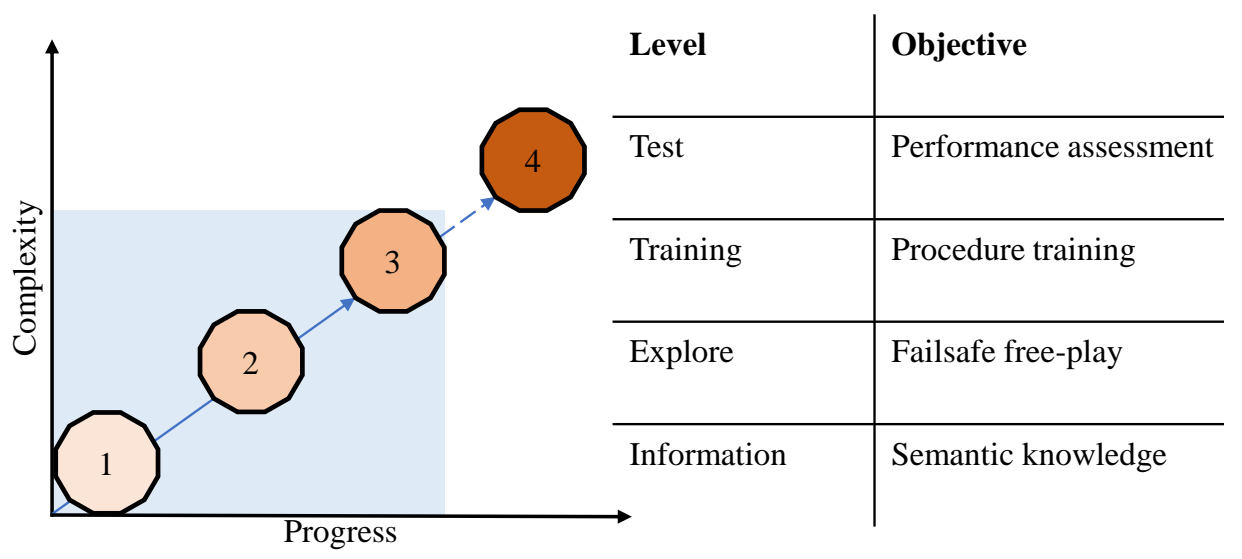

Figure 5. The different complexity level of the exercise

When activating the exercise in the CBS, a popup window appears with the selection of the four complexity levels.

- The Information scenario was programmed with descriptive text in popup windows appearing each time a component or system function was activated by the trainee, providing both component information, normal parameter values and functionality in the system.

- The Explore scenario was programmed with no information, goals, or support, with the purpose of testing the systems functionality and components interaction in a condition with impunity from error or faults.

- The Training scenario, the core of the exercise, was programmed with instruction to perform the pre-departure procedure. A narrative set of popup windows advised trainees on the system status, provided positive feedback upon completion of procedural steps, provided correctional instruction on errors or inadequate parameters, and gave ques and reminders throughout the exercise. Once the exercise was completed and exited correctly, the CBS provided the trainee an assessment with a score of their performance. The goal of the assessment was to motivate repeated practice until (1) a 
error-free 220-point score was obtained or (2) a score obtained which the trainee was confident in or contempt with, to proceed to the test scenario alternative.

- The Test scenario gave trainees instruction to perform the same pre-departure procedure, but without any supportive structures, and provided an assessment with the same 220-piont scale as in the training alternative. For each attempt, the exercise was programmed to record and store simulator metrics and assessment scores. The score from the final training attempt and the test was collected for analysis.

\section{$\underline{\text { Data Collection and Analysis }}$}

Each phase of the procedure resulted in data collected:

- The knowledge test after the knowledge acquisition phase probed important elements from the lectured content by 10 multiple-choice items. The test was only available with one attempt.

- Training scores from the skill-acquisition phase was recorded according to the programmed metrics of the simulation.

- After the Test scenario the trainees inscribed an online 7-point Likert questionnaire with the Self-efficacy for Learning and Performance (SELP) scale of the Motivated Strategies for Self-regulatory Learning Questionnaire (MSLQ) (Duncan et al., 2015). The 8-items of the SELP were calculated to a factor average to capture the level of self-efficacy related to the training. Test scores according to the programmed simulator metrics was recorded.

The quasi-experimental within-group design has no control group. Collected data was analysed in SPSS 26.0.

\section{Results}

The paper's research question, "How can CBS adapt to individual training needs?", tested two hypotheses: $\left(\mathrm{H}_{1}\right)$ "knowledge prior to training will be significant to the final training performance" and $\left(\mathrm{H}_{2}\right)$ "the level of self-efficacy will positively predict the final training performance".

The knowledge test resulted in $M D=8.670, S D=1.495$ in a range up to 10 possible correct responses. The sample $(N=18)$ was not normally distributed as the Shapiro-Wilk resulted in $W=0.184$ and $p=0.002$.

The final training scores resulted in $M D=163.33, S D=59.606$ (out of a possible 220-point score). The sample $(N=18)$ was not normally distributed as the Shapiro-Wilk resulted in $W=0.827, p=0.004$. The coefficient of variation was $C V=0.365$.

The Self-Efficacy for Learning and Performance (SLEP) factor score resulted in $M D=4.729$, $S D=0.966$. The sample $(N=18)$ was found to be normally distributed with the Shapiro-Wilk test reporting $W=0.924, p=0.152$. The dataset was found to have good internal consistency, with a Cronbach`s alpha $\alpha=0.873$. 
The test scores resulted in $M D=183.611, S D=39.400$ (out of a possible 220-point score). The sample $(N=18)$ was not normally distributed as the Shapiro-Wilk resulted in $W=0.803$, $p=0.002$. The coefficient of variation was $C V=0.214$.

Testing the hypothesis $\mathrm{H}_{1}$ was performed by measuring the correlation between the knowledge test and the final training scores. A Spearman`s rho test resulted in $r_{S}=-0.039, p=0.878$. This suggests that there is no correlation between the knowledge test and the final training score, and thus rejection of hypothesis 1 .

Testing hypothesis $\mathrm{H}_{2}$ was performed by measuring the correlation between the level of selfefficacy and the final training score. A Spearman`s rho resulted in $r_{S}=0.471, p=0.048$. This suggests that there is a significant correlation between the level of self-efficacy and the final training score. Second, a linear regression tested the prediction of the final training score based on SELP and found $F(1,16)=5.323, p=0.035, r^{2}=0.250$. A univariate analysis of variance was then produced to provide a F-test for heteroskedasticity of the residuals, which found an insignificant relationship $F(1,16)=.794, p=.389$. Further, distribution of residuals contained no outliers and hold a close to normal distribution according to the PP plot. The regression model suggests a significant prediction, thus acceptance of hypothesis 2 .

\section{Discussion}

The statistical analysis found no significant correlation between the knowledge test and the final training score, and thus hypothesis 1 was rejected. A similar result was found by Chernikova et al. (2020) which states that prior knowledge is expected to hold large influence over learning, however the effect of simulation is greater when compared. The two measures capture two different outcome constructs at different stages in the overall process, as by the definition of Kraiger et al. (1993). A measure after a knowledge-acquisition phase, with a uniform time dissipation and a standardized delivery, will be prone to the influence of trainee characteristics, i.e., individual differences. The declarative knowledge development exceeds through the skill-acquisition phase, i.e., the simulator training, as declarative knowledge is transformed into procedural knowledge. Through the training process, general system knowledge is applied to the specific task and the trainees keep aggregating new comprehension to their knowledge foundation.

Hypothesis 2 was accepted, as self-efficacy was able to predict the training scores. Selfefficacy develops in connection to the training process (Kraiger et al., 1993), as chronologically it exists before the training start and develops during, between and after the repeated training. This empowers the trainee's development towards better performance as increased selfefficacy provide resilience to the difficulties encountered during training (Ford et al., 1998). As each training scenario attempt gives a metric performance assessment, the perception of one`s own capability to perform should be free from false expectancy. The hypothesized relation between self-efficacy and the final training score proved significant, arguing for a contextual development through multiple training attempts. During this skill-acquisition phase, proceduralization of knowledge occurs as knowledge is applied to succeed the task procedure and approximate a satisfying level of goal completion. As there was no extrinsic pressure to train repeatedly or to which level of proficiency was considered sufficient, all performance was at the discretion of the trainee. Chernikova et al. (2020) challenges future research to identify effective types and sequences of scaffolding in simulation-based learning with a focus on trainees at different levels of prior knowledge and experience. Feedback at different levels was utilized to capture the initial engagement of trainees with different level of prior knowledge, to 
keep engagement and facilitate development during the training. Hattie and Timperley (2016) states that both providing and receiving feedback requires much skill by the instructor and trainees.

The descriptive data of the final training score and the test score show some interesting trends. At the point of self-evaluating their final training score, the trainees decided to conclude their training and proceed to the test. The mean difference in scores increased from the training scenario to the test scenario. Considering that the training scenario had an automated system feedback and ques throughout the exercise procedure while the test scenario was without any such supportive structures, a logical assumption would be to expect no mean difference or a lower test scenario performance if the trainee was not ready to be assessed. However, the higher training scenario score reveals that there are effects of the training that was not captured by the training scenario measurements, i.e., learning is a process that also occurs beyond the training scenario. Furthermore, the central tendency reduces towards the test scores as evident with the standard deviations. In standardized terms of the coefficient of variation, the standard deviation of the final training scores is $36,5 \%$ of its mean and the standard deviation of the test scores is $21,4 \%$ of its mean, with a larger mean score than in the former condition. These phenomena argue for the effectiveness of the training, as would be expected in repeated learning- or training processes (Bell et al., 2017). Early proficiency, as with prior knowledge, is thus not a good predictor of the training outcome as a sufficient training design is able to mitigate trainee characteristics that creates individual learning curves (Bell et al., 2017; Kraiger et al., 1993).

Decentralized simulator training, as explored in this paper, offers some effects that on-site simulators may not. Results indicate that CBS is an operable technology for its purpose, although training delivery as designed for this study it is not likely to be a substitution to onsite simulators. The software might be the same between CBS and on-site simulators, however the CBS is fundamentally more reliant on objective automated assessment, where in comparison, training with on-site simulators traditionally utilize more prone to subjective assessment methodologies of the present instructor. The different training delivery conditions should rather be explored in an intertwined design along with lecture-based learning, to exploit beneficial effects from all conditions of learning, and thus find the balance between objective and subjective evaluation as requested by the STCW (2017). The training condition of this study addresses a relatively simple procedural task with a training design that leverages feedback at different levels and task complexity at different levels. Although tailored to the specific task while operationalized, this approach can be applied to a variety of scenarios in MET.

Considering the programming of exercises to a varied target population it is recommended to create simple and decomposed tasks rather than more encompassing scenarios for a uniform population with high prior knowledge, at least when working with comparatively novice students. To implement asynchronous CBS training as applied in this study, CBS training should be organized as a supplement to the traditional on-site simulator training or lecture programme, as an individual repetitive session over an adequate period. To retain control over how the automated feedback is received and thus the effect of it, the training programme can be designed with one or more synchronous sessions, either individually or collectively, also providing direct feedback at the correct level according to Hattie and Timperley (2016). An individual synchronous session would imply the instructor and trainee to interact through live video conference while the trainee is casting the simulator, preferably at a point of stagnation in the training progress. A collective synchronous session would require an instructor to 
interact real-time with a group of multimedia-distributed trainees, while casting the simulator and lecturing during the task prosecution.

\section{$\underline{\text { Limitations }}$}

The sample $(N=18)$ were recruited with different programme and institutional affiliations in nautical sciences at college level $(n=11)$, nautical sciences at university level $(n=1)$, marine engineering at college level $(n=1)$, and marine engineering at university level $(n=5)$. A larger sample would allow group discrimination for between-group comparisons and better generalization to other populations, e.g., other cohorts.

The sample size raises some statistical concern where the robustness of the tests used, and the resulting effect sizes need to be addressed. The effect of the regression model categorizes as large, which should be favourable of a fit model considering the sample size, although the sample is small in absolute terms (Field, 2009) there is no clear violation of the assumptions for a regression model. Sample size also influence the accuracy of correlations, where simulations have proposed substantially larger samples before confidence in the estimate is established (Schönbrodt \& Perugini, 2013). The sample size and the effect sizes give the H2 correlation and regression a $1-\beta$ power of .657 and .633 , respectively, according to G*Power. This probability of falsely rejecting the null hypothesis mainly derive from the sample size. Similarly, the external validity of this paper's result may be low due to the sample size, however Hackshaw (2008) notes that small samples should be sufficient in hypothesis-generating studies if statistical power is attained. The Norwegian Universities and Colleges Admission Service (2020) can inform that a total of 588 students who started the first semester in these aforementioned education programmes in 2020. The actual number of active students at the second semester is not controlled for dropout at the time of the study, but the Database for Statistics on Higher Education (2020) indicate a 9,2\% dropout across all maritime studies between these semesters for this cohort. If the sample can represent any population is an issue of judgement, where this study's sample is an 3,4\% extraction of the full national cohort.

The knowledge test could have been expanded with more items to constitute two merged scales, one addressing the content of the lectures and one addressing the procedure. Such an instrument could have been administered pre- and post-treatment to capture the change in declarative knowledge. The self-efficacy instrument could also have been administered early in the training to capture false expectancy and the consolidation of self-perception through the training. These two propositions would provide additional measures that could further describe training effectiveness and the individually adaptive features of the training design.

\section{Conclusion}

This paper investigates Cloud-Based Simulators can adapt to individual training and offers some reflective inspiration for the MET educator. It is apparent that individually adaptive training is possible with current CBS technology, yet it remains to the MET community to establish what approaches to take. A research-based approach will facilitate application of CBS that leverages constructs deemed as positive to the learning process, such as self-efficacy. The preliminary research-based evidence of this paper could provide MET educators inspiration for initiating decentralized simulator training for novice trainees; however, a larger confirmatory study is necessary to be able to generalize results. Future contributions from the authors of this paper will explore the positive effects of CBS and to whom these effects apply. 


\section{$\underline{\text { Acknowledgements }}$}

The authors thank Kongsberg Digital for their contribution to this study by providing licenses for this research on the K-Sim Engine MAN 6S70ME-C SCC simulator through their K-Sim Connect platform.

\section{References}

Bell, B., I. Tannenbaum, S., Ford, J., Noe, R., \& Kraiger, K. (2017). 100 Years of Training and Development Research: What We Know and Where We Should Go (Vol. 102). https://doi.org/10.1037/ap10000142

Chernikova, O., Heitzmann, N., Stadler, M., Holzberger, D., Seidel, T., \& Fischer, F. (2020). Simulation-Based Learning in Higher Education: A Meta-Analysis [Article]. Review of educational research, 90(4), 499-541, Article 0034654320933544. https://doi.org/10.3102/0034654320933544

Database for Statistics on Higher Education. (2020). https://dbh.nsd.uib.no/

Duncan, T., pintrich, p., smith, d., \& McKeachie, W. (2015). Motivated Strategies for Learning Questionnaire (MSLQ) Manual. https://doi.org/10.13140/RG.2.1.2547.6968

Field, A. (2009). Discovering statistics using SPSS : (and sex and drugs and rock ' $n$ ' roll) (3rd ed. ed.). SAGE.

Ford, J. K., Smith, E. M., Weissbein, D. A., Gully, S. M., \& Salas, E. (1998). Relationships of goal orientation, metacognitive activity, and practice strategies with learning outcomes and transfer. Journal of Applied Psychology, 83(2), 218-233. https://doi.org/10.1037/0021$\underline{9010.83 .2 .218}$

Hackshaw, A. (2008). Small studies: strengths and limitations. Eur Respir J, 32(5), 1141-1143. https://doi.org/10.1183/09031936.00136408

Hattie, J., \& Timperley, H. (2016). The Power of Feedback. Review of educational research, 77(1), 81-112. https://doi.org/10.3102/003465430298487

STCW; International Convention on Standards of Training, Certification and Watchkeeping for Seafarers; Including 2010 Manila amendments; STCW Convention and STCW Code, STCW 2017 (2017).

Kraiger, K., Ford, J. K., \& Salas, E. (1993). Application of cognitive, skill-based, and affective theories of learning outcomes to new methods of training evaluation. Journal of Applied Psychology, 78(2), 311-328. https://doi.org/10.1037/0021-9010.78.2.311

Kulhavy, R. W. (1977). Feedback in Written Instruction. Review of educational research, 47(2). https://doi.org/10.3102/00346543047002211

Mallam, S. C., Nazir, S., \& Renganayagalu, S. K. (2019). Rethinking Maritime Education, Training, and Operations in the Digital Era: Applications for Emerging Immersive Technologies. Journal of Marine Science and Engineering, 7(12). https://doi.org/10.3390/jmse7120428

Schönbrodt, F. D., \& Perugini, M. (2013). At what sample size do correlations stabilize? Journal of Research in Personality, 47(5), 609-612. https://doi.org/10.1016/j.jrp.2013.05.009

Smith, J., Doody, K., \& Veitch, B. (2019). Being prepared for emergencies: a virtual environment experiment on the retention and maintenance of egress skills. WMU Journal of Maritime Affairs, 18(3), 425-449. https://doi.org/10.1007/s13437-019-00174-y

The Norwegian Universities and Colleges Admission Service. (2020). https://www.samordnaopptak.no/info/english/

Zimmerman, B. J. (2008). Investigating Self-Regulation and Motivation: Historical Background, Methodological Developments, and Future Prospects [Article]. American Educational Research Journal, 45(1), 166-183. https://doi.org/10.3102/0002831207312909 